\title{
Tensor Product of Massey Products
}

\author{
Qi Bing ZHENG \\ Department of Mathematics, Nankai University, Tianjin 300071, P. R. China \\ E-mail: zhengqb@nankai.edu.cn
}

\begin{abstract}
In this paper, we interpret Massey products in terms of realizations (twitsting cochains) of certain differential graded coalgebras with values in differential graded algebras. In the case where the target algebra is the cobar construction of a differential graded commutative Hopf algebra, we construct the tensor product of realizations and show that the tensor product is strictly associative, and commutative up to homotopy.
\end{abstract}

Keywords Realization, Hopf algebra

MR(2000) Subject Classification 55, 18G

\section{Realizations on DGA}

Throughout this paper, $F$ always denotes a given field, $\otimes$ means $\otimes_{F}$. All objects of categories in this paper are connected bigraded vector spaces over $F$ of finite type, that is, if $V$ is a bigraded vector space, then $V^{s, t}$ is a finite dimensional space for every fixed $s, t \geq 0$, and $V^{s, t}=0$, otherwise. Obviously, the tensor product of two such vector spaces still satisfies these conditions. We always use "|.|" to denote the first degree of vector spaces and "\|l||" to denote the second degree. For single-graded vector space $V$, we regard it bigraded by letting $|v|=0$ for all $v \in V$. We first give some basic definitions.

A coalgebra means a connected, coassociative coalgebra over $F$. That is, if $V$ is a coalgebra, then there is an augmentation $\varepsilon: V \rightarrow F$ with a diagonal map $\Delta: V \rightarrow V \otimes V$ such that $(\varepsilon \otimes 1) \Delta=\Delta(1 \otimes \varepsilon)=1$ and $(1 \otimes \Delta) \Delta=(\Delta \otimes 1) \Delta$. We denote $\bar{V}=$ ker $\varepsilon$, then the above property means that $\Delta(1)=1 \otimes 1$ and for all $v \in \bar{V}, \Delta(v)=1 \otimes v+v \otimes 1+\sum v^{\prime} \otimes v^{\prime \prime}$, where $v^{\prime}, v^{\prime \prime} \in \bar{V}$. This means that we have uniquely defined the reduced diagonal map $\bar{\Delta}: \bar{V} \rightarrow \bar{V} \otimes \bar{V}$ defined by $\bar{\Delta}(v)=\Delta(v)-1 \otimes v-v \otimes 1$ for all $v \in \bar{V}$. It is easy to check that $(1 \otimes \bar{\Delta}) \bar{\Delta}=(\bar{\Delta} \otimes 1) \bar{\Delta}$. Thus, we may inductively define $f^{(r)}=\left(1 \otimes f^{(r-1)}\right) f=\left(f^{(r-1)} \otimes 1\right) f$ for $f=\Delta$ and $\bar{\Delta}$.

For two coalgebras $V_{1}$ and $V_{2}$, the tensor product coalgebra $V_{1} \otimes V_{2}$ 's diagonal map is defined by $\Delta\left(v_{1} \otimes v_{2}\right)=\sum(-1)^{\left\langle v_{1}^{\prime \prime}, v_{2}^{\prime}\right\rangle} v_{1}^{\prime} v_{2}^{\prime} \otimes v_{1}^{\prime \prime} v_{2}^{\prime \prime}$ for all $v_{i} \in V_{i}$ and $\Delta\left(v_{i}\right)=\sum v_{i}^{\prime} \otimes v_{i}^{\prime \prime}(\langle a, b\rangle=$ $|a||b|+\|a\|\|b\|)$.

If $D$ is a DGA (cochain complex), then it is a bigraded algebra over $F$ with unit and there is a differential $d$ on $D$ such that for all $a, b \in D, d(a b)=(d a) b+(-1)^{|a|} a(d b)$. This implies that the bidegree of the differential is $(1,0)$, i.e. $|d a|=|a|+1$ and $\|d a\|=\|a\|$ for all $a \in D$. A (right) DGA-module $M$ over $D$ is a chain complex that is a (right) module over $D$ and $d(m a)=(d m) a+(-1)^{|m|} m(d a)$ for all $m \in M$ and $a \in D$.

Definition 1.1 Let $V$ be a coalgebra and $D$ a DGA. A realization of $V$ on $D$ is a linear map $\pi: \bar{V}^{s, t} \rightarrow D^{s+1, t}$ such that for all $v \in \bar{V}$, if $\bar{\Delta}(v)=\sum v^{\prime} \otimes v^{\prime \prime}$, then $d \pi(v)=$ $\sum(-1)^{\left|v^{\prime}\right|+1} \pi\left(v^{\prime}\right) \pi\left(v^{\prime \prime}\right)$.

The obstruction of realizations is a generalization of Massey-product. We define Massey coalgebra $M_{n}(n>0)$ as follows. $\bar{M}_{n}$ has a basis $\left\{e_{i, j} \mid 1 \leq i<j \leq n+1\right\}$, the diagonal map is 
defined by $\left(e_{i, i}=1\right) \Delta\left(e_{i, j}\right)=\sum_{s=i}^{j-1} e_{i, s} \otimes e_{s+1, j}$. The bidegree of $M_{n}$ satisfies that $\left|e_{i, j}\right|+1=$ $\left|e_{i, s}\right|+\left|e_{s, j}\right|$ and $\left\|e_{i, j}\right\|=\left\|e_{i, s}\right\|+\left\|e_{s, j}\right\|$ for all $1 \leq i<s<j \leq n$. Then that a realization $\pi: \bar{M}_{n} \rightarrow D$ exists implies that a given Massey-product $\left\langle\pi\left(e_{1,2}\right), \ldots, \pi\left(e_{n, n+1}\right)\right\rangle$ is defined and cohomologous to 0 in $D(n>1)$. If $\left\langle\pi\left(e_{1,2}\right), \ldots, \pi\left(e_{n, n+1}\right)\right\rangle \nsim 0$, but it is defined, then it is the obstruction for $\pi\left(e_{1, n+1}\right)$ to be defined. Thus, the more we know about realizations, the more kinds of multi-product we will have. The purpose of this paper is to study the structure of realizations.

Definition 1.2 Suppose $M$ is a DGA module over the DGA $D$ and $\pi: \bar{V} \rightarrow D$ is a realization of coalgebra $V$ on $D$. For $a \in M$, da=0, we define Massey product $\langle a ; \pi(v)\rangle$ as follows. If $v$ is primitive $(\bar{\Delta}(v)=0)$, we define $\langle a ; \pi(v)\rangle=(-1)^{|a|} a \pi(v)$ and for non-primitive $v$ with $\bar{\Delta}(v)=\sum v^{\prime} \otimes v^{\prime \prime}$, a Massey product $\langle a ; \pi(v)\rangle$ is defined if for all $v^{\prime},\left\langle a ; \pi\left(v^{\prime}\right)\right\rangle$ is defined and cohomologous to 0 in $M$ and we define

$$
\langle a ; \pi(v)\rangle=(-1)^{|a|} a \pi(v)+\sum(-1)^{|a|+\left|v^{\prime}\right|} \overline{\left\langle a ; \pi\left(v^{\prime}\right)\right\rangle} \pi\left(v^{\prime \prime}\right),
$$

where $\overline{d\left\langle a ; \pi\left(v^{\prime}\right)\right\rangle}=\left\langle a ; \pi\left(v^{\prime}\right)\right\rangle$. It is obvious that when $v$ is not primitive, the product is not uniquely defined and we use $\operatorname{In}\langle a ; \pi(v)\rangle$ to denote the whole class of elements.

Definition 1.3 For two realizations $\pi_{1}, \pi_{2}$ of the coalgebra $V$ on $D$, we say $\pi_{1}$ is homotopic to $\pi_{2}$ (denoted by $\pi_{1} \sim \pi_{2}$ ) if there is a homotopy $T: \bar{V}^{s, t} \rightarrow D^{s, t}$ such that for all $v \in \bar{V}$, $\bar{\Delta}(v)=\sum v^{\prime} \otimes v^{\prime \prime}$,

$$
d T(v)+\sum \pi_{1}\left(v^{\prime}\right) T\left(v^{\prime \prime}\right)+\sum(-1)^{\left|v^{\prime}\right|+1} T\left(v^{\prime}\right) \pi_{2}\left(v^{\prime \prime}\right)=\pi_{1}(v)-\pi_{2}(v) .
$$

Theorem 1.1 The homotopy relation defined above is an equivalence relation.

Proof To prove $\pi \sim \pi$, we take the homotopy $T=0(T(v)=0$ for all $v \in \bar{V})$. Suppose $T_{1}$ is a homotopy from $\pi_{1}$ to $\pi_{2}$ and $T_{2}$ is a homotopy from $\pi_{2}$ to $\pi_{3}$. Then we define the composite homotopy $T_{2} T_{1}$ by

$$
\left(T_{2} T_{1}\right)(v)=T_{1}(v)-\sum T_{1}\left(v^{\prime}\right) T_{2}\left(v^{\prime \prime}\right)+T_{2}(v)
$$

for all $v \in \bar{V}$ with $\bar{\Delta}(v)=\sum v^{\prime} \otimes v^{\prime \prime}$. It is a direct checking that $T_{2} T_{1}$ is a homotopy from $\pi_{1}$ to $\pi_{3}$. For $T$ a homotopy from $\pi_{1}$ to $\pi_{2}$, the equalities (for all $v \in \bar{V}$ )

$$
T(v)-\sum T\left(v^{\prime}\right) T^{-1}\left(v^{\prime \prime}\right)+T^{-1}(v)=0
$$

uniquely determine a map $T^{-1}$. It is easy to check that this $T^{-1}$ is a homotopy from $\pi_{2}$ to $\pi_{1}$ and we call it the inverse of $T$. Therefore, " " is an equivalence relation.

Theorem 1.2 Let $\pi_{1}, \pi_{2}$ be two homotopic realizations of the coalgebra $V$ on the DGA $D$ and $M$ a DGA-module over $D$. Then for $a \in M, d a=0$, and $v \in \bar{V}$, a Massey product $\left\langle a ; \pi_{1}(v)\right\rangle$ exists if and only if a Massey product $\left\langle a ; \pi_{2}(v)\right\rangle$ exists such that $\left\langle a ; \pi_{1}(v)\right\rangle \sim\left\langle a ; \pi_{2}(v)\right\rangle$, that is, $\operatorname{In}\left\langle a ; \pi_{1}(v)\right\rangle \sim \operatorname{In}\left\langle a ; \pi_{2}(v)\right\rangle$.

Proof We take $d(a T(v))=\left\langle a ; \pi_{1}(v)\right\rangle-\left\langle a ; \pi_{2}(v)\right\rangle$.

\section{Tensor Product}

In this section, we only study realizations on the cobar complex of a Hopf algebra, so throughout this section, $H$ always denotes a given graded commutative and coassociative Hopf algebra. That is, $H$ is both a coalgebra and a commutative algebra such that the product map is a coalgebra homomorphism from $H \otimes H$ to $H$ and for all $a, b \in H, a b=(-1)^{\|a\|\|b\|} b a$. By our convention, $H$ is bigraded by defining $|h|=0$ for all $h \in H$.

For $H$, the cobar complex $C(H)$ is a DGA defined as follows. As an algebra, $C(H)$ is the algebra freely generated by $\bar{H}$. That is, $C(H)$ has a basis consisting of 1 and elements of the form $\left[v_{1}\left|v_{2}\right| \cdots \mid v_{n}\right]$ with $v_{i} \in \bar{H}$. The bidegree of $C(V)$ is defined by that $\left|\left[v_{1}|\cdots| v_{n}\right]\right|=n$ and $\left\|\left[v_{1}|\cdots| v_{n}\right]\right\|=\sum_{i=1}^{n}\left\|v_{i}\right\|$. The differential on $C(H)$ is defined by

$$
d\left[v_{1}\left|v_{2}\right| \cdots \mid v_{n}\right]=\sum_{i=1}^{n} \sum(-1)^{i}\left[v_{1}|\cdots| v_{i}^{\prime}\left|v_{i}^{\prime \prime}\right| \cdots \mid v_{n}\right],
$$


where $\bar{\Delta}\left(v_{i}\right)=\sum v_{i}^{\prime} \otimes v_{i}^{\prime \prime}$. It is obvious that $C(H)$ is a DGA. To distinguish the product of $H$ from that of $C(H)$, we always use "|" to denote the product of cobar complex.

Notice that if $\pi$ is a realization of the coalgebra $V$ on the cobar complex $C(H)$, then for $v \in \bar{V}, \pi(v)$ may not be of the form $[w]$ with $w \in \bar{H}$. That is, $\pi(v)$ may be linear combinations of elements of the form $\left[w_{1}|\cdots| w_{n}\right]$ with $w_{i} \in \bar{H}$ such that $|v|+1=n$. Therefore, $V$ must be bigraded.

To describe linear maps on $C(H)$, we have to recall the definition of relative signs in [1]. For bigraded symbols $x_{1}, x_{2}, \ldots, x_{n}$, if $\left(i_{1}, i_{2}, \ldots, i_{n}\right)$ is a re-ordering of $(1,2, \ldots, n)$, then the sign of $\left(x_{i_{1}}, x_{i_{2}}, \ldots, x_{i_{n}}\right)$ relative to $\left(x_{1}, x_{2}, \ldots, x_{n}\right)$, or the sign of the permutation $\left(\begin{array}{cccc}x_{i_{1}}, & x_{i_{2}}, & \ldots, & x_{i_{n}} \\ x_{1}, & x_{2}, & \ldots, & x_{n}\end{array}\right)$ is defined as follows. The sign of $\left(x_{1}, \ldots, x_{i}, x_{i+1}, \ldots, x_{n}\right)$ relative to $\left(x_{1}, \ldots, x_{i+1}, x_{i}, \ldots, x_{n}\right)$ is $(-1)^{\left\langle x_{i}, x_{i+1}\right\rangle}(\langle x, y\rangle=|x||y|+\|x\|\|y\|)$. The relative sign of the composite of two permutations is the product of the relative signs of the two permutations. Then, according to the proof (of $\mu_{f}$ ) in Section 1 of [1], the relative sign is well-defined.

Theorem 2.1 Let $V$ be a coalgebra and $\pi$ a realization of $V$ on $C(H)$. Then for all $v \in V$, there is a linear map $\pi(v ;): C(H)^{s, t} \rightarrow C(H)^{s+|v|, t+\|v\|}$ satisfying that for all $a \in C(H)$, $\pi(1 ; a)=a$ and for $v \in \bar{V}, \bar{\Delta}(v)=\sum v^{\prime} \otimes v^{\prime \prime}$,

$$
\begin{aligned}
d \pi(v ; a)+(-1)^{|v|+1} \pi(v ; d a)= & (-1)^{\left|v^{\prime}\right|+1} \pi\left(v^{\prime}\right)\left|\pi\left(v^{\prime \prime} ; a\right)+(-1)^{\left\langle a, v^{\prime \prime}\right\rangle+|a|+|v|} \pi\left(v^{\prime} ; a\right)\right| \pi\left(v^{\prime \prime}\right) \\
& +(-1)^{|v|+1}[\pi(v), a],
\end{aligned}
$$

where $\langle x, y\rangle=|x||y|+\|x\|\|y\|$ and $[x, y]=x\left|y-(-1)^{\langle x, y\rangle} y\right| x$.

Proof For $u=\left[u_{1}|\cdots| u_{n}\right]\left(u_{i} \in \bar{H}\right), h \in \bar{H}$, we define $u * 1=u, 1 * 1=1,1 * h=[h]$, and

$$
u * h=\sum(-1)^{\mu}\left[u_{1} h^{(1)}\left|u_{2} h^{(2)}\right| \cdots \mid u_{n} h^{(n)}\right],
$$

where $\Delta^{(n-1)}(h)=\sum h^{(1)} \otimes h^{(2)} \otimes \cdots h^{(n)}$ and $(-1)^{\mu}$ is the sign of $\left(\left[u_{1}\right], h^{(1)}, \ldots,\left[u_{n}\right], h^{(n)}\right)$ relative to $\left(\left[u_{1}\right], \ldots,\left[u_{n}\right], h^{(1)}, \ldots, h^{(n)}\right)$ (Notice the difference of bidegrees between $[u]$ and $u$ ). It is a direct checking that the $*$-product satisfies

$$
d(u * h)-(d u) * h-(-1)^{|u|}[u,[h]]=\sum(-1)^{|u|}\left(u * h^{\prime}\right)\left|\left[h^{\prime \prime}\right]-\sum(-1)^{\left\langle u, h^{\prime}\right\rangle}\left[h^{\prime}\right]\right|\left(u * h^{\prime \prime}\right),
$$

where $\bar{\Delta}(h)=\sum h^{\prime} \otimes h^{\prime \prime}$. So, we may inductively define

$1 \pi(v ; 1)=0$ and for all $h \in \bar{H}, \pi(v ;[h])=\pi(v) * h$.

2 For all $a, b \in C(H)$ and $v \in V, \Delta(v)=1 \otimes v+v \otimes 1+\sum v^{\prime} \otimes v^{\prime \prime}$,

$$
\pi(v ; a \mid b)=\pi(v ; a)\left|b+(-1)^{\langle a, v\rangle} a\right| \pi(v ; b)+\sum(-1)^{\left\langle a, v^{\prime \prime}\right\rangle} \pi\left(v^{\prime} ; a\right) \mid \pi\left(v^{\prime \prime} ; b\right) .
$$

Then, the proof is just a direct checking.

There may be other linear maps satisfying the properties of $\pi(v ;)$ in the theorem.

Theorem 2.2 Let $V_{1}, V_{2} \in \mathscr{V}$ and $\pi_{i}: \bar{V}_{i} \rightarrow C(H)(i=1,2)$ be two realizations. Then, there is a tensor product realization $\left(\pi_{1} \otimes \pi_{2}\right): \overline{V_{1} \otimes V_{2}} \rightarrow C(H)$ defined as follows. For all $v_{i} \in \bar{V}_{i}(i=1,2),\left(\pi_{1} \otimes \pi_{2}\right)\left(v_{1} \otimes 1\right)=\pi_{1}\left(v_{1}\right),\left(\pi_{1} \otimes \pi_{2}\right)\left(1 \otimes v_{2}\right)=\pi_{2}\left(v_{2}\right),\left(\pi_{1} \otimes \pi_{2}\right)\left(v_{1} \otimes v_{2}\right)=$ $\pi_{1}\left(v_{1} ; \pi_{2}\left(v_{2}\right)\right)$, where $\pi_{1}\left(v_{1} ;\right)$ is as defined in the previous theorem. The tensor product of realizations is associative, that is, $\left(\pi_{1} \otimes \pi_{2}\right) \otimes \pi_{3}=\pi_{1} \otimes\left(\pi_{2} \otimes \pi_{3}\right)$.

Proof It is obvious that $\pi_{1} \otimes \pi_{2}$ is a realization on such elements $v_{1} \otimes 1$ and $1 \otimes v_{2}$ with $v_{i} \in \bar{V}_{i}, i=1,2$. Now suppose $\bar{\Delta}\left(v_{i}\right)=\sum v_{i}^{\prime} \otimes v_{i}^{\prime \prime}$; then

$$
\begin{aligned}
d\left(\left[v_{1} \otimes v_{2}\right]\right)= & (-1)^{\left|v_{1}\right|+1}\left[v_{1} \otimes 1 \mid 1 \otimes v_{2}\right]+(-1)^{\left\langle v_{1}, v_{2}\right\rangle+\left|v_{2}\right|+1}\left[1 \otimes v_{2} \mid v_{1} \otimes 1\right] \\
& +\sum\left((-1)^{\left|v_{1}\right|+\left|v_{2}^{\prime}\right|+1}\left[v_{1} \otimes v_{2}^{\prime} \mid 1 \otimes v_{2}^{\prime \prime}\right]+(-1)^{\left\langle v_{1}, v_{2}^{\prime}\right\rangle+\left|v_{2}^{\prime}\right|+1}\left[1 \otimes v_{2}^{\prime} \mid v_{1} \otimes v_{2}^{\prime \prime}\right]\right) \\
& +\sum\left((-1)^{\left|v_{1}^{\prime}\right|+1}\left[v_{1}^{\prime} \otimes 1 \mid v_{1}^{\prime \prime} \otimes v_{2}\right]+(-1)^{\left\langle v_{1}^{\prime \prime}, v_{2}\right\rangle+\left|v_{1}^{\prime}\right|+\left|v_{2}\right|+1}\left[v_{1}^{\prime} \otimes v_{2} \mid v_{1}^{\prime \prime} \otimes 1\right]\right) \\
& +\sum(-1)^{\left\langle v_{1}^{\prime \prime}, v_{2}^{\prime}\right\rangle+\left|v_{1}^{\prime}\right|+\left|v_{2}^{\prime}\right|+1}\left[v_{1}^{\prime} \otimes v_{2}^{\prime} \mid v_{1}^{\prime \prime} \otimes v_{2}^{\prime \prime}\right]
\end{aligned}
$$


and by Theorem 2.1,

$$
\begin{aligned}
d\left(\pi_{1} \otimes \pi_{2}\right)\left(v_{1} \otimes v_{2}\right)= & d \pi\left(v_{1} ; \pi\left(v_{2}\right)\right) \\
= & (-1)^{\left|v_{1}\right|} \pi_{1}\left(v_{1} ; \sum(-1)^{\left|v_{2}^{\prime}\right|+1} \pi_{2}\left(v_{2}^{\prime}\right) \mid \pi\left(v_{2}^{\prime \prime}\right)\right) \\
& +(-1)^{\left|v_{1}^{\prime}\right|+1} \pi_{1}\left(v_{1}^{\prime}\right) \mid \pi_{1}\left(v_{1}^{\prime \prime} ; \pi_{2}\left(v_{2}\right)\right) \\
& +(-1)^{\left\langle v_{1}^{\prime \prime}, v_{2}\right\rangle+\left|v_{1}^{\prime}\right|+\left|v_{2}\right|+1} \pi_{1}\left(v_{1}^{\prime} ; \pi_{2}\left(v_{2}\right)\right) \mid \pi_{1}\left(v_{1}^{\prime \prime}\right) \\
& +(-1)^{\left|v_{1}\right|+1}\left[\pi_{1}\left(v_{1}\right), \pi_{2}\left(v_{2}\right)\right] \\
= & \left.\sum(-1)^{\left|v_{1}\right|+\left|v_{2}^{\prime}\right|+1} \pi_{1}\left(v_{1} ; \pi_{2}\left(v_{2}^{\prime}\right)\right) \mid \pi_{2}\left(v_{2}^{\prime \prime}\right)\right) \\
& +\sum(-1)^{\left\langle v_{1}, v_{2}^{\prime}\right\rangle+\left|v_{2}^{\prime}\right|+1} \pi_{2}\left(v_{2}^{\prime}\right) \mid \pi_{1}\left(v_{1} ; \pi_{2}\left(v_{2}^{\prime \prime}\right)\right) \\
& +\sum(-1)^{\left\langle v_{1}^{\prime \prime}, v_{2}^{\prime}\right\rangle+\left|v_{1}^{\prime}\right|+\left|v_{2}^{\prime}\right|+1} \pi_{1}\left(v_{1}^{\prime} ; \pi_{2}\left(v_{2}^{\prime}\right)\right) \mid \pi_{1}\left(v_{1}^{\prime \prime} ; \pi_{2}\left(v_{2}^{\prime \prime}\right)\right) \\
& +\sum(-1)^{\left|v_{1}^{\prime}\right|+1} \pi_{1}\left(v_{1}^{\prime}\right) \mid \pi_{1}\left(v_{1}^{\prime \prime} ; \pi_{2}\left(v_{2}\right)\right) \\
& +\sum(-1)^{\left\langle v_{1}^{\prime \prime}, v_{2}\right\rangle+\left|v_{1}^{\prime}\right|+\left|v_{2}\right|+1} \pi_{1}\left(v_{1}^{\prime} ; \pi_{2}\left(v_{2}\right)\right) \mid \pi_{1}\left(v_{1}^{\prime \prime}\right) \\
& +(-1)^{\left|v_{1}\right|+1} \pi_{1}\left(v_{1}\right)\left|\pi_{2}\left(v_{2}\right)+(-1)^{\left\langle v_{1}, v_{2}\right\rangle+\left|v_{2}\right|+1} \pi_{2}\left(v_{2}\right)\right| \pi_{1}\left(v_{1}\right) \\
= & \left(\pi_{1} \otimes \pi_{2}\right)_{*}\left(d\left[v_{1} \otimes v_{2}\right]\right) \quad\left(\left(\pi_{1} \otimes \pi_{2}\right)_{*}(a \mid b)=\left(\pi_{1} \otimes \pi_{2}\right)(a) \mid\left(\pi_{1} \otimes \pi_{2}\right)(b)\right) .
\end{aligned}
$$

Thus, $\pi_{1} \otimes \pi_{2}$ is a realization.

To prove the associativity of the tensor product, we need only to prove that for all $v_{1} \in \bar{V}_{1}$, $v_{2} \in \bar{V}_{2}$ and $a \in C(H)$,

$$
\left(\pi_{1} \otimes \pi_{2}\right)\left(v_{1} \otimes v_{2} ; a\right)=\pi_{1}\left(v_{1} ; \pi_{2}\left(v_{2} ; a\right)\right) .
$$

Suppose $a=\left[h_{1}|\cdots| h_{n}\right]\left(h_{i} \in \bar{H}\right), \Delta^{(n-1)}\left(v_{i}\right)=\sum v_{i}^{(1)} \otimes \cdots \otimes v_{i}^{(n)}, i=1,2$. Then by Theorem 2.1, we have that

$$
\left(\pi_{1} \otimes \pi_{2}\right)\left(v_{1} \otimes v_{2} ; a\right)=\sum(-1)^{\mu}\left(\pi_{1} \otimes \pi_{2}\right)\left(v_{1}^{(1)} \otimes v_{2}^{(1)} ;\left[h_{1}\right]\right)|\cdots|\left(\pi_{1} \otimes \pi_{2}\right)\left(v_{1}^{(n)} \otimes v_{2}^{(n)} ;\left[h_{n}\right]\right),
$$

where $(-1)^{\mu}$ is the sign of $\left(v_{1}^{(1)}, v_{2}^{(1)},\left[h_{1}\right], \ldots, v_{1}^{(n)}, v_{2}^{(n)},\left[h_{n}\right]\right)$ relative to $\left(v_{1}^{(1)}, \ldots, v_{1}^{(n)}, v_{2}^{(1)}, \ldots\right.$, $\left.v_{2}^{(n)},\left[h_{1}\right], \ldots,\left[h_{n}\right]\right)$. Similarly,

$$
\begin{aligned}
\pi_{1}\left(v_{1} ; \pi_{2}\left(v_{2} ; a\right)\right) & =\pi_{1}\left(v_{1} ; \sum(-1)^{\mu^{\prime}} \pi_{2}\left(v_{2}^{(1)} ;\left[h_{1}\right]\right)|\cdots| \pi_{2}\left(v_{2}^{(n)} ;\left[h_{n}\right]\right)\right) \\
& =\sum(-1)^{\mu} \pi_{1}\left(v_{1}^{(1)} ; \pi_{2}\left(v_{2}^{(1)} ;\left[h_{1}\right]\right)\right)|\cdots| \pi_{1}\left(v_{1}^{(n)} ; \pi_{2}\left(v_{2}^{(n)} ;\left[h_{n}\right]\right)\right),
\end{aligned}
$$

where $(-1)^{\mu}$ is as above and $(-1)^{\mu^{\prime}}$ is the sign of $\left(v_{2}^{(1)},\left[h_{1}\right], \ldots, v_{2}^{(n)},\left[h_{n}\right]\right)$ relative to $\left(v_{2}^{(1)}, \ldots\right.$, $\left.v_{2}^{(n)},\left[h_{1}\right], \ldots,\left[h_{n}\right]\right)$. So we need only to prove that for all $h \in \bar{H}$,

$$
\left(\pi_{1} \otimes \pi_{2}\right)\left(v_{1} \otimes v_{2} ;[h]\right)=\pi_{1}\left(v_{1} ; \pi_{2}\left(v_{2} ;[h]\right)\right) .
$$

Suppose $\pi_{2}\left(v_{2}\right)=\left[u_{1}|\cdots| u_{n}\right]\left(u_{i} \in \bar{H}\right), \Delta^{(n-1)}(h)=\sum h^{(1)} \otimes \cdots \otimes h^{(n)}$. Then

$$
\left.\pi_{2}\left(v_{2} ; h\right]\right)=\sum(-1)^{\mu_{1}}\left[u_{1} h^{(1)}\left|u_{2} h^{(2)}\right| \cdots \mid u_{n} h^{(n)}\right],
$$

where $(-1)^{\mu_{1}}$ is the sign of $\left(\left[u_{1}\right], h^{(1)}, \ldots,\left[u_{n}\right], h^{(n)}\right)$ relative to $\left(\left[u_{1}\right], \ldots,\left[u_{n}\right], h^{(1)}, \ldots, h^{(n)}\right)$. So by Theorem 2.1,

$$
\pi_{1}\left(v_{1} ; \pi_{2}\left(v_{2} ;[h]\right)\right)=\sum(-1)^{\mu_{2}} \pi_{1}\left(v_{1}^{(1)}\right) *\left(u_{1} h^{(1)}\right)|\cdots| \pi_{1}\left(v_{1}^{(n)}\right) *\left(u_{n} h^{(n)}\right),
$$

where $(-1)^{\mu_{2}}$ is the sign of $\left(v_{1}^{(1)},\left[u_{1}\right], h^{(1)}, \ldots, v_{1}^{(n)},\left[u_{n}\right], h^{(n)}\right)$ relative to $\left(v_{1}^{(1)}, \ldots, v_{1}^{(n)},\left[u_{1}\right], \ldots\right.$, $\left.\left[u_{n}\right], h^{(1)}, \ldots, h^{(n)}\right)$. Similarly,

$$
\pi_{1}\left(v_{1} ; \pi_{2}\left(v_{2}\right)\right)=\sum(-1)^{\mu_{3}} \pi_{1}\left(v_{1}^{(1)}\right) * u_{1}|\cdots| \pi_{1}\left(v_{1}^{(n)}\right) * u_{n},
$$


where $(-1)^{\mu_{3}}$ is the sign of $\left(v_{1}^{(1)},\left[u_{1}\right], \ldots, v_{1}^{(n)},\left[u_{n}\right]\right)$ relative to $\left(v_{1}^{(1)}, \ldots, v_{1}^{(n)},\left[u_{1}\right], \ldots,\left[u_{n}\right]\right)$. So

$$
\begin{aligned}
\left(\pi_{1} \otimes \pi_{2}\right)\left(v_{1} \otimes v_{2} ;[h]\right) & =\pi_{1}\left(v_{1} ; \pi_{2}\left(v_{2}\right)\right) * h \\
& =\sum(-1)^{\mu_{3}}\left(\pi_{1}\left(v_{1}^{(1)}\right) * u_{1}|\cdots| \pi_{1}\left(v_{1}^{(n)}\right) * u_{n}\right) * h \\
& =\sum(-1)^{\mu_{2}} \pi_{1}\left(v_{1}^{(1)}\right) *\left(u_{1} h^{(1)}\right)|\cdots| \pi_{1}\left(v_{1}^{(n)}\right) *\left(u_{n} h^{(n)}\right) \\
& =\pi_{1}\left(v_{1} ; \pi_{2}\left(v_{2} ;[h]\right)\right) .
\end{aligned}
$$

The theorem is proved.

Theorem 2.3 For $i=1, \ldots, n, V_{i}$ is a coalgebra and $\pi_{i}, \pi_{i}^{\prime}: \bar{V}_{i} \rightarrow C(H)$ are homotopic realizations. Then, we have $\pi_{1} \otimes \cdots \otimes \pi_{n} \sim \pi_{1}^{\prime} \otimes \cdots \otimes \pi_{n}^{\prime}$.

Proof We need only to prove the following special case. Let $\pi: \bar{V} \rightarrow C(H)$ and $\pi_{1}, \pi_{1}^{\prime}: \bar{V}_{1} \rightarrow$ $C(H)$ be realizations such that $\pi_{1} \sim \pi_{1}^{\prime}$ with homotopy $T$. Then $\pi \otimes \pi_{1} \sim \pi \otimes \pi_{1}^{\prime}$ and $\pi_{1} \otimes \pi \sim \pi_{1}^{\prime} \otimes \pi$. Define $T \otimes 1: \overline{V_{1} \otimes V} \rightarrow C(H)$ by $(T \otimes 1)(1 \otimes v)=0,(T \otimes 1)\left(v_{1} \otimes v\right)=0$, $(T \otimes 1)\left(v_{1} \otimes 1\right)=T\left(v_{1}\right)$ for all $v_{1} \in \bar{V}_{1}$ and $v \in \bar{V}$. Then it is easy to check that $T \otimes 1$ is a homotopy from $\pi_{1} \otimes \pi$ to $\pi_{1}^{\prime} \otimes \pi$. Define $1 \otimes T: \overline{V \otimes V_{1}} \rightarrow C(H)$ by $(1 \otimes T)(v \otimes 1)=0$, $(1 \otimes T)\left(v \otimes v_{1}\right)=\pi\left(v ; T\left(v_{1}\right)\right),(1 \otimes T)\left(1 \otimes v_{1}\right)=T\left(v_{1}\right)$ for all $v \in \bar{V}$ and $v_{1} \in \bar{V}_{1}$. Then it is easy to check that $1 \otimes T$ is a homotopy from $\pi \otimes \pi_{1}$ to $\pi \otimes \pi_{1}^{\prime}$.

Theorem 2.4 For $i=1,2$, let $\pi_{i}: \bar{V}_{i} \rightarrow C(H)$ be two realizations. Then

$$
\pi_{1} \otimes \pi_{2} \sim(-1)^{\left\langle\pi_{1}, \pi_{2}\right\rangle} \pi_{2} \otimes \pi_{1},
$$

where for all $v_{i} \in \bar{V}_{i},(-1)^{\left\langle\pi_{1}, \pi_{2}\right\rangle}\left(\pi_{2} \otimes \pi_{1}\right)\left(v_{1} \otimes v_{2}\right)=(-1)^{\left\langle v_{1}, v_{2}\right\rangle} \pi_{2}\left(v_{2} ; \pi\left(v_{1}\right)\right)$.

The proof of the theorem is quite complicated and needs a lot of preliminaries. We first define some maps. Since we will often compute elements of $\bar{H}$ and $C(H)$ in one formula, we use $\bar{u}, \bar{v}, \ldots$ to denote elements of $\bar{H}$ and use $u, v, \ldots$ to denote elements of $C(H)$.

We define bilinear maps $S, S^{\prime}: C(H) \otimes C(H) \rightarrow C(H)$ as follows. For any $u, v \in C(H)$, $S(u ; 1)=S(1 ; v)=S^{\prime}(u ; 1)=S^{\prime}(1 ; v)=0$, and

$$
S(u ; v)=\sum(-1)^{\left\langle u-1, v_{1}\right\rangle} v_{1}\left|u * \bar{v}_{2}\right| v_{3}, \quad S^{\prime}(u ; v)=\sum(-1)^{|u|+\left\langle v-1,\left[\bar{u}_{2}\right] \mid u_{3}\right\rangle} u_{1}\left|v * \bar{u}_{2}\right| u_{3},
$$

where the sum is taken throughout all possible $u_{1}, \bar{u}_{2}, u_{3}$ and $v_{1}, \bar{v}_{2}, v_{3}$ such that $u=u_{1}\left|\left[\bar{u}_{2}\right]\right| u_{3}$, $v=v_{1}\left|\left[\bar{v}_{2}\right]\right| v_{3}$ with $\bar{u}_{2}, \bar{v}_{2} \in \bar{H}$ and $x-1$ is a bigraded symbol such that $|x-1|=|x|-1$, $\|x-1\|=\|x\|$.

We inductively define multi-linear maps $S, S^{\prime}$ as follows. For any $u, v, w_{i} \in C(H), i=$ $1,2, \ldots, n, n>1$,

$$
\begin{aligned}
& S\left(w_{1}, \ldots, w_{n} ; v\right)=\sum(-1)^{\left\langle w_{n}-1, v_{1}\right\rangle} S\left(w_{1}, \ldots, w_{n-1} ; v_{1}\right)\left|w_{n} * \bar{v}_{2}\right| v_{3}, \\
& S^{\prime}\left(u ; w_{1}, \ldots, w_{n}\right)=\sum(-1)^{\left|u_{1}\right|+\left|\bar{u}_{2}\right|+1+\left\langle w_{1}-1,\left[\bar{u}_{2}\right] \mid u_{3}\right\rangle} u_{1}\left|w_{1} * \bar{u}_{2}\right| S^{\prime}\left(u_{3} ; w_{2}, \ldots, w_{n}\right),
\end{aligned}
$$

where the sum and symbol $x-1$ are just as above.

We define bilinear map $S_{2}: C(H) \otimes C(H) \rightarrow C(H)$ as follows. For any $u, v \in C(H)$, if $u=[\bar{u}]$ or $v=[\bar{v}]$ with $\bar{u}, \bar{v} \in \bar{H}$, then $S_{2}(u ; v)=0$; otherwise

$$
S_{2}(u ; v)=\sum(-1)^{|u|+\left\langle v_{1}-1,\left[\bar{u}_{2}\right] \mid u_{3}\right\rangle} u_{1}\left|v_{1} * \bar{u}_{2}\right| u_{3} * \bar{v}_{2} \mid v_{3},
$$

where the sum and symbol $x-1$ are just as above.

We inductively define multi-linear maps $S_{2}$ as follows. For any $u, v, w_{i} \in C(H), i=$ $1,2, \ldots, n, n>1$,

$$
\begin{aligned}
& S_{2}\left(w_{1}, \ldots, w_{n} ; v\right)=\sum(-1)^{\left\langle w_{n}-1, v_{1}\right\rangle} S_{2}\left(w_{1}, \ldots, w_{n-1} ; v_{1}\right)\left|w_{n} * \bar{v}_{2}\right| v_{3}, \\
& S_{2}\left(u ; w_{1}, \ldots, w_{n}\right)=\sum(-1)^{\left|u_{1}\right|+\left|\bar{u}_{2}\right|+1+\left\langle w_{1}-1,\left[\bar{u}_{2}\right] \mid u_{3}\right\rangle} u_{1}\left|w_{1} * \bar{u}_{2}\right| S_{2}\left(u_{3} ; w_{2}, \ldots, w_{n}\right),
\end{aligned}
$$

where the sum and symbol $x-1$ are just as above. 
For $u_{i}, v_{j}, \in C(H), i=1,2, \ldots, m, j=1,2, \ldots, n, m, n>1$,

$$
\begin{aligned}
& S_{2}\left(u_{1}, \ldots, u_{m} ; v_{1}, \ldots, v_{n}\right) \\
& \quad=\sum(-1)^{\mu+\left|u_{1,1}\right|+\left|\bar{u}_{1,2}\right|+1} u_{1,1}\left|v_{1} * \bar{u}_{1,2}\right| S_{2}\left(u_{1,3}, \ldots, u_{m-1} ; v_{2}, \ldots, v_{n, 1}\right)\left|u_{m} * \bar{v}_{n, 2}\right| v_{n, 3},
\end{aligned}
$$

where the sum is taken throughout all possible $u_{1,1}, \bar{u}_{1,2}, u_{1,3}$ and $v_{n, 1}, \bar{v}_{n, 2}, v_{n, 3}$ such that $u_{1}=u_{1,1}\left|\left[\bar{u}_{1,2}\right]\right| u_{1,3}, v_{n}=v_{n, 1}\left|\left[\bar{v}_{n, 2}\right]\right| v_{n, 3}$ with $\bar{u}_{1,2}, \bar{v}_{1,2} \in \bar{H}$ and $(-1)^{\mu}$ is the sign of $\left(v_{1}-\right.$ $\left.1,\left[\bar{u}_{1,2}\right], u_{1,3}, u_{2}-1, \ldots, u_{m-1}-1, v_{2}-1, \ldots, v_{n-1}-1, v_{n, 1}, u_{m}-1\right)$ relative to $\left(\left[\bar{u}_{1,2}\right], u_{1,3}, u_{2}-\right.$ $\left.1, \ldots, u_{m-1}-1, u_{m}-1, v_{1}-1, v_{2}-1, \ldots, v_{n-1}-1, v_{n, 1}\right)(x-1$ as above).

Lemma 2.1 Let $S, S^{\prime}, S_{2}$ be as above. Then we have

$1 d S_{2}(u ; v)+S_{2}(d u ; v)+(-1)^{|u|-1} S_{2}(u ; d v)=S(u ; v)-S^{\prime}(u ; v)$.

2 For $m>1$,

$$
\begin{aligned}
d S_{2}\left(u_{1}, \ldots, u_{m} ; v\right)+ & \sum_{s=1}^{m}(-1)^{\left|\mu_{s-1}\right|} S_{2}\left(u_{1}, \ldots, d u_{s}, \ldots, u_{m} ; v\right)+(-1)^{\left|\mu_{m}\right|} S_{2}\left(u_{1}, \ldots, u_{m} ; d v\right) \\
& +\sum_{s=1}^{m-1}(-1)^{\left|\mu_{s}\right|} S_{2}\left(u_{1}, \ldots, u_{s} \mid u_{s+1}, \ldots, u_{m} ; v\right)+u_{1} \mid S_{2}\left(u_{2}, \ldots, u_{m} ; v\right) \\
& -(-1)^{\left\langle u_{m}-1, v-1\right\rangle+\left|\mu_{m-1}\right|+|v-1|} S_{2}\left(u_{1}, \ldots, u_{m-1} ; v\right) \mid u_{m} \\
= & S\left(u_{1}, \ldots, u_{m} ; v\right),
\end{aligned}
$$

where $\mu_{s}$ is a bigraded symbol such that $\left|\mu_{s}\right|=\left|u_{1}\right|+\cdots+\left|u_{s}\right|-s,\left\|\mu_{s}\right\|=\left\|u_{1}\right\|+\cdots+\left\|u_{s}\right\|$ and $x-1$ is a bigraded symbol such that $|x-1|=|x|-1,\|x-1\|=\|x\|$.

3 For $n>1$,

$$
\begin{aligned}
d S_{2}\left(u ; v_{1}, \ldots, v_{n}\right)+S_{2}\left(d u ; v_{1}, \ldots, v_{n}\right)+\sum_{t=1}^{n}(-1)^{|u-1|+\left|\tau_{t-1}\right|} S_{2}\left(u ; v_{1}, \ldots, d v_{t}, \ldots, v_{n}\right) \\
\quad+\sum_{t=1}^{n-1}(-1)^{|u-1|+\left|\tau_{t}\right|} S_{2}\left(u ; v_{1}, \ldots, v_{t} \mid v_{t+1}, \ldots, v_{n}\right)+(-1)^{\left\langle u-1, v_{1}-1\right\rangle} v_{1} \mid S_{2}\left(u ; v_{2}, \ldots, v_{n}\right) \\
\quad-(-1)^{|u-1|+\left|\tau_{n-1}\right|} S_{2}\left(u ; v_{1}, \ldots, v_{n-1}\right) \mid v_{n} \\
=-S^{\prime}\left(u ; v_{1}, \ldots, v_{n}\right),
\end{aligned}
$$

where $\tau_{t}$ is a bigraded symbol such that $\left|\tau_{t}\right|=\left|v_{1}\right|+\cdots+\left|v_{t}\right|-t,\left\|\tau_{t}\right\|=\left\|v_{1}\right\|+\cdots+\left\|v_{t}\right\|$ and $x-1$ is as in $\mathbf{2}$.

4 For $m>1, n>1$,

$$
\begin{aligned}
d S_{2} & \left(u_{1}, \ldots, u_{m} ; v_{1}, \ldots, v_{n}\right) \quad \text { (a) } \\
& +\sum_{s=1}^{m}(-1)^{\left|\mu_{s-1}\right|} S_{2}\left(u_{1}, \ldots, d u_{s}, \ldots, u_{m} ; v_{1}, \ldots, v_{n}\right) \quad \text { (b) } \\
& +\sum_{t=1}^{n}(-1)^{\left|\mu_{m}\right|+\left|\tau_{t-1}\right|} S_{2}\left(u_{1}, \ldots, u_{m} ; v_{1}, \ldots, d v_{t}, \ldots, v_{n}\right) \quad(\mathrm{c}) \\
& +\sum_{s=1}^{m-1}(-1)^{\left|\mu_{s}\right|} S_{2}\left(u_{1}, \ldots, u_{s} \mid u_{s+1}, \ldots, u_{m} ; v_{1}, \ldots, v_{n}\right) \quad(\mathrm{d}) \\
& +\sum_{t=1}^{n-1}(-1)^{\left|\mu_{m}\right|+\left|\tau_{t}\right|} S_{2}\left(u_{1}, \ldots, u_{m} ; v_{1}, \ldots, v_{t} \mid v_{t+1}, \ldots, v_{n}\right) \quad(\mathrm{e}) \\
& +u_{1} \mid S_{2}\left(u_{2}, \ldots, u_{m} ; v_{1}, \ldots, v_{n}\right) \quad(\mathrm{f}) \\
& -(-1)^{\left\langle u_{m}-1, \tau_{n}\right\rangle+\left|\mu_{m-1}\right|+\left|\tau_{n}\right|} S_{2}\left(u_{1}, \ldots, u_{m-1} ; v_{1}, \ldots, v_{n}\right) \mid u_{m} \\
& +(-1)^{\left\langle\mu_{m}, v_{1}-1\right\rangle} v_{1} \mid S_{2}\left(u_{1}, \ldots, u_{m} ; v_{2}, \ldots, v_{n}\right) \quad(\mathrm{h})
\end{aligned}
$$




$$
\begin{aligned}
& -(-1)^{\left|\mu_{m}\right|+\left|\tau_{n-1}\right|} S_{2}\left(u_{1}, \ldots, u_{m} ; v_{1}, \ldots, v_{n-1}\right) \mid v_{n} \\
& +\sum_{t=1}^{n-1}(-1)^{\left\langle\tilde{\mu}_{1}, \tau_{t}\right\rangle} S^{\prime}\left(u_{1} ; v_{1}, \ldots, v_{t}\right) \mid S_{2}\left(u_{2}, \ldots, u_{m} ; v_{t+1}, \ldots, v_{n}\right) \quad(\mathrm{j}) \\
& -\sum_{s=1}^{m-1}(-1)^{\left\langle\tilde{\mu}_{s}, \tau_{n-1}\right\rangle+\left|\mu_{s}\right|+\left|\tau_{n-1}\right|} S_{2}\left(u_{1}, \ldots, u_{s} ; v_{1} ; \cdots, v_{n-1}\right) \mid S\left(u_{s+1}, \ldots, u_{m} ; v_{n}\right) \\
= & 0,
\end{aligned}
$$

where $\mu_{s}$ and $\tau_{t}$ are as in $\mathbf{2}$ and $\mathbf{3}$ and $\left|\tilde{\mu}_{s}\right|=\left|\mu_{m}\right|-\left|\mu_{s}\right|,\left\|\tilde{\mu}_{s}\right\|=\left\|\mu_{m}\right\|-\left\|\mu_{s}\right\|$.

Proof Notice that the Steenrod cohomology operation can also be generalized to bigraded Hopf algebras. Let $W$ be the free resolution of the group of integers modular 2 , and $\theta: W \otimes C(H) \otimes$ $C(H) \rightarrow C(H)$ be the group module morphism defined in [2]. Then, we have $\theta\left(e_{1} \otimes u \otimes v\right)=$ $(-1)^{|u|} S(u ; v), \theta\left(e_{2} \otimes u \otimes v\right)=(-1)^{|u|} S_{2}(u ; v), S^{\prime}(u ; v)=(-1)^{\langle u, v\rangle} S(v ; u)$. So 1 holds. We only prove $\mathbf{4}$ of the theorem and omit the proof of $\mathbf{2}$ and $\mathbf{3}$, since it is an easier copy of the proof of 4.

To prove 4, we use double induction on $m$ and $n$. If $m=n=2$, it is a direct checking. Now suppose 4 holds for $m<k$ and $n<l$ with at least on of $k$ and $l$ is $>2$. Then for $m=k$ and $n=l$, we compute the left side of 4 . To simplify the formula, we omit all the sum symbol " $\sum$ " and all signs $(-1)^{*}$ and so denote $(-1)^{*} x$ simply by $x$. The checking of the signs of the proof is an easy but tedious work and we omit it. In the following computation, the (omitted) sum is always taken throughout all possible $u_{1,1}, \bar{u}_{1,2}, u_{1,3}$ and $v_{n, 1}, \bar{v}_{n, 2}, v_{n, 3}$ such that $u_{1}=u_{1,1}\left|\left[\bar{u}_{1,2}\right]\right| u_{1,3}, v_{n}=v_{n, 1}\left|\left[\bar{v}_{n, 2}\right]\right| v_{n, 3}$ with $\bar{u}_{1,2}, \bar{v}_{n, 2} \in \bar{H}$ and $\bar{\Delta}\left(\bar{u}_{1,2}\right)=\bar{u}_{1,2}^{\prime} \otimes \bar{u}_{1,2}^{\prime \prime}$, $\bar{\Delta}\left(\bar{v}_{n, 2}\right)=\bar{v}_{n, 2}^{\prime} \otimes \bar{v}_{n, 2}^{\prime \prime}$, and we have

$$
\begin{aligned}
& \text { (a) }=\left(d u_{1,1}\right)\left|v_{1} * \bar{u}_{1,2}\right| S_{2}\left(u_{1,3}, \ldots, u_{m-1} ; v_{2}, \ldots, v_{n, 1}\right)\left|u_{m} * \bar{v}_{n, 2}\right| v_{n, 3} \\
& +u_{1,1}\left|\left(d v_{1}\right) * \bar{u}_{1,2}\right| S_{2}\left(u_{1,3}, \ldots, u_{m-1} ; v_{2}, \ldots, v_{n, 1}\right)\left|u_{m} * \bar{v}_{n, 2}\right| v_{n, 3} \\
& +u_{1,1}\left|v_{1} * \bar{u}_{1,2}^{\prime}\right|\left[\bar{u}_{1,2}^{\prime \prime}\right]\left|S_{2}\left(u_{1,3}, \ldots, u_{m-1} ; v_{2}, \ldots, v_{n, 1}\right)\right| u_{m} * \bar{v}_{n, 2} \mid v_{n, 3} \\
& +u_{1,1}\left|\left[\bar{u}_{1,2}^{\prime}\right]\right| v_{1} * \bar{u}_{1,2}^{\prime \prime}\left|S_{2}\left(u_{1,3}, \ldots, u_{m-1} ; v_{2}, \ldots, v_{n, 1}\right)\right| u_{m} * \bar{v}_{n, 2} \mid v_{n, 3} \\
& +u_{1,1}\left|v_{1}\right|\left[\bar{u}_{1,2}\right]\left|S_{2}\left(u_{1,3}, \ldots, u_{m-1} ; v_{2}, \ldots, v_{n, 1}\right)\right| u_{m} * \bar{v}_{n, 2} \mid v_{n, 3} \\
& +u_{1,1}\left|\left[\bar{u}_{1,2}\right]\right| v_{1}\left|S_{2}\left(u_{1,3}, \ldots, u_{m-1} ; v_{2}, \ldots, v_{n, 1}\right)\right| u_{m} * \bar{v}_{n, 2} \mid v_{n, 3} \\
& +u_{1,1}\left|v_{1} * \bar{u}_{1,2}\right| d S_{2}\left(u_{1,3}, \ldots, u_{m-1} ; v_{2}, \ldots, v_{n, 1}\right)\left|u_{m} * \bar{v}_{n, 2}\right| v_{n, 3} \\
& +u_{1,1}\left|v_{1} * \bar{u}_{1,2}\right| S_{2}\left(u_{1,3}, \ldots, u_{m-1} ; v_{2}, \ldots, v_{n, 1}\right)\left|\left(d u_{m}\right) * \bar{v}_{n, 2}\right| v_{n, 3} \\
& +u_{1,1}\left|v_{1} * \bar{u}_{1,2}\right| S_{2}\left(u_{1,3}, \ldots, u_{m-1} ; v_{2}, \ldots, v_{n, 1}\right)\left|u_{m} * \bar{v}_{n, 2}^{\prime}\right|\left[\bar{v}_{n, 2}^{\prime \prime}\right] \mid v_{n, 3} \\
& +u_{1,1}\left|v_{1} * \bar{u}_{1,2}\right| S_{2}\left(u_{1,3}, \ldots, u_{m-1} ; v_{2}, \ldots, v_{n, 1}\right)\left|\left[\bar{v}_{n, 2}^{\prime}\right]\right| u_{m} * \bar{v}_{n, 2}^{\prime \prime} \mid v_{n, 3} \\
& +u_{1,1}\left|v_{1} * \bar{u}_{1,2}\right| S_{2}\left(u_{1,3}, \ldots, u_{m-1} ; v_{2}, \ldots, v_{n, 1}\right)\left|u_{m}\right|\left[\bar{v}_{n, 2}\right] \mid v_{n, 3} \\
& +u_{1,1}\left|v_{1} * \bar{u}_{1,2}\right| S_{2}\left(u_{1,3}, \ldots, u_{m-1} ; v_{2}, \ldots, v_{n, 1}\right)\left|\left[\bar{v}_{n, 2}\right]\right| u_{m} \mid v_{n, 3} \\
& +u_{1,1}\left|v_{1} * \bar{u}_{1,2}\right| S_{2}\left(u_{1,3}, \ldots, u_{m-1} ; v_{2}, \ldots, v_{n, 1}\right)\left|u_{m} * \bar{v}_{n, 2}\right|\left(d v_{n, 3}\right) . \\
& =\left(d u_{1,1}\right)\left|v_{1} * \bar{u}_{1,2}\right| S_{2}\left(u_{1,3}, \ldots, u_{m-1} ; v_{2}, \ldots, v_{n, 1}\right)\left|u_{m} * \bar{v}_{n, 2}\right| v_{n, 3} \\
& +u_{1,1}\left|\left[\bar{u}_{1,2}^{\prime}\right]\right| v_{1} * \bar{u}_{1,2}^{\prime \prime}\left|S_{2}\left(u_{1,3}, \ldots, u_{m-1} ; v_{2}, \ldots, v_{n, 1}\right)\right| u_{m} * \bar{v}_{n, 2} \mid v_{n, 3} \\
& +u_{1,1}\left|v_{1} * \bar{u}_{1,2}^{\prime}\right| S_{2}\left(\left[\bar{u}_{1,2}^{\prime \prime}\right] \mid u_{1,3}, \ldots, u_{m-1} ; v_{2}, \ldots, v_{n, 1}\right)\left|u_{m} * \bar{v}_{n, 2}\right| v_{n, 3} \\
& +u_{1,1}\left|v_{1} * \bar{u}_{1,2}\right| S_{2}\left(u_{1,3}, \ldots, d u_{s}, \ldots, u_{m-1} ; v_{2}, \ldots, v_{n, 1}\right)\left|u_{m} * \bar{v}_{n, 2}\right| v_{n, 3} \\
& +u_{1,1}\left|v_{1} * \bar{u}_{1,2}\right| S_{2}\left(u_{1,3}, \ldots, u_{m-1} ; v_{2}, \ldots, v_{n, 1}\right)\left|\left(d u_{m}\right) * \bar{v}_{n, 2}\right| v_{n, 3} \\
& +u_{1,1}\left|\left(d v_{1}\right) * \bar{u}_{1,2}\right| S_{2}\left(u_{1,3}, \ldots, u_{m-1} ; v_{2}, \ldots, v_{n, 1}\right)\left|u_{m} * \bar{v}_{n, 2}\right| v_{n, 3} \\
& +u_{1,1}\left|v_{1} * \bar{u}_{1,2}\right| S_{2}\left(u_{1,3}, \ldots, u_{m-1} ; v_{2}, \ldots, d v_{t}, \ldots, v_{n, 1}\right)\left|u_{m} * \bar{v}_{n, 2}\right| v_{n, 3} \\
& +u_{1,1}\left|v_{1} * \bar{u}_{1,2}\right| S_{2}\left(u_{1,3}, \ldots, u_{m-1} ; v_{2}, \ldots, v_{n, 1} \mid\left[\bar{v}_{n, 2}^{\prime}\right]\right)\left|u_{m} * \bar{v}_{n, 2}^{\prime \prime}\right| v_{n, 3}
\end{aligned}
$$




$$
\begin{aligned}
& +u_{1,1}\left|v_{1} * \bar{u}_{1,2}\right| S_{2}\left(u_{1,3}, \ldots, u_{m-1} ; v_{2}, \ldots, v_{n, 1}\right)\left|u_{m} * \bar{v}_{n, 2}^{\prime}\right|\left[\bar{v}_{n, 2}^{\prime \prime}\right] \mid v_{n, 3} \\
& +u_{1,1}\left|v_{1} * \bar{u}_{1,2}\right| S_{2}\left(u_{1,3}, \ldots, u_{m-1} ; v_{2}, \ldots, v_{n, 1}\right)\left|u_{m} * \bar{v}_{n, 2}\right|\left(d v_{n, 3}\right) \text {. } \\
& \text { (d) }+(\mathrm{e}) \\
& =u_{1} \mid S_{2}\left(u_{2}, \ldots, u_{m} ; v_{1}, \ldots, v_{n}\right) \\
& +u_{1,1}\left|v_{1} * \bar{u}_{1,2}\right| S_{2}\left(u_{1,3}, \ldots, u_{s} \mid u_{s+1}, \ldots, u_{m-1} ; v_{2}, \ldots, v_{n, 1}\right)\left|u_{m} * \bar{v}_{n, 2}\right| v_{n, 3} \\
& +u_{1,1}\left|v_{1} * \bar{u}_{1,2}\right| S_{2}\left(u_{1,3}, \ldots, \ldots, u_{m-2} ; v_{2}, \ldots, v_{n, 1}\right)\left|u_{m-1}\right| u_{m} * \bar{v}_{n, 2} \mid v_{n, 3} \\
& +u_{1,1}\left|v_{1} * \bar{u}_{1,2}\right| S_{2}\left(u_{1,3}, \ldots, \ldots, u_{m-2} ; v_{2}, \ldots, v_{n, 1}\right)\left|u_{m-1} * \bar{v}_{n, 2}\right| u_{m} \mid v_{n, 3} \\
& +u_{1,1}\left|v_{1} * \bar{u}_{1,2}\right| S_{2}\left(u_{1,3}, \ldots, \ldots, u_{m-2} ; v_{2}, \ldots, v_{n, 1}\right)\left|u_{m-1} * \bar{v}_{n, 2}^{\prime}\right| u_{m} * \bar{v}_{n, 2}^{\prime \prime} \mid v_{n, 3} \\
& +u_{1,1}\left|v_{1}\right| v_{2} * \bar{u}_{1,2}\left|S_{2}\left(u_{1,3}, \ldots, u_{m-1} ; v_{3}, \ldots, v_{n, 1}\right)\right| u_{m} * \bar{v}_{n, 2} \mid v_{n, 3} \\
& +u_{1,1}\left|v_{1} * \bar{u}_{1,2}\right| v_{2}\left|S_{2}\left(u_{1,3}, \ldots, u_{m-1} ; v_{3}, \ldots, v_{n, 1}\right)\right| u_{m} * \bar{v}_{n, 2} \mid v_{n, 3} \\
& +u_{1,1}\left|v_{1} * \bar{u}_{1,2}^{\prime}\right| v_{2} * \bar{u}_{1,2}^{\prime \prime}\left|S_{2}\left(u_{1,3}, \ldots, u_{m-1} ; v_{3}, \ldots, v_{n, 1}\right)\right| u_{m} * \bar{v}_{n, 2} \mid v_{n, 3} \\
& +u_{1,1}\left|v_{1} * \bar{u}_{1,2}\right| S_{2}\left(u_{1,3}, \ldots, u_{m-1} ; v_{2}, \ldots, v_{t} \mid v_{t+1}, \ldots, v_{n, 1}\right)\left|u_{m} * \bar{v}_{n, 2}\right| v_{n, 3} \\
& +S_{2}\left(u_{1}, \ldots, u_{m} ; v_{1}, \ldots, v_{n-1}\right) \mid v_{n} . \\
& \text { (f) }+(\mathrm{g})+(\mathrm{h})+(\mathrm{i}) \\
& =u_{1} \mid S_{2}\left(u_{2}, \ldots, u_{m} ; v_{1}, \ldots, v_{n}\right) \\
& +S_{2}\left(u_{1}, \ldots, u_{m-1} ; v_{1}, \ldots, v_{n}\right) \mid u_{m} \\
& +v_{1} \mid S_{2}\left(u_{1}, \ldots, u_{m} ; v_{2}, \ldots, v_{n}\right) \quad(36) \\
& +S_{2}\left(u_{1}, \ldots, u_{m} ; v_{1}, \ldots, v_{n-1}\right) \mid v_{n} \text {. } \\
& =u_{1,1}\left|v_{1} * \bar{u}_{1,2}\right| S^{\prime}\left(u_{1,3} ; v_{2}, \ldots, v_{t}\right) \mid S_{2}\left(u_{2}, \ldots, u_{m} ; v_{t+1}, \ldots, v_{n}\right) \quad \text { (38) } \\
& +S_{2}\left(u_{1}, \ldots, u_{s} ; v_{1}, \ldots, v_{n-1}\right)\left|S\left(u_{s+1}, \ldots, u_{m-1} ; v_{n, 1}\right)\right| u_{m} * \bar{v}_{n, 2} \mid v_{n, 3} \\
& +u_{1,1}\left|v_{1} * \bar{u}_{1,2}\right| u_{1,3} \mid S_{2}\left(u_{2}, \ldots, u_{m} ; v_{2}, \ldots, v_{n}\right) \\
& +S_{2}\left(u_{1}, \ldots, u_{m-1} ; v_{1}, \ldots, v_{n-1}\right)\left|v_{n, 1}\right| u_{m} * \bar{v}_{n, 2} \mid v_{n, 3} \text {. }
\end{aligned}
$$

It is obvious that the following sum is 0 .

$(1)+(14),(2)+(19),(4)+(15),(8)+(18),(9)+(22),(13)+(23),(24)+(34),(33)+(37)$. Since we also have

$$
\begin{aligned}
S_{2}\left([\bar{u}] \mid u_{1}, \ldots, u_{m} ; v_{1}, \ldots, v_{n}\right)= & (-1)^{|\bar{u}|+1}[\bar{u}] \mid S_{2}\left(u_{1}, \ldots, u_{m} ; v_{1}, \ldots, v_{n}\right) \\
& +\sum(-1)^{\mu^{\prime}+|\bar{u}|+1} v_{1} * \bar{u} \mid S_{2}\left(u_{1}, \ldots, u_{m} ; v_{2}, \ldots, v_{n}\right), \\
S_{2}\left(u_{1}, \ldots, u_{m} ; v_{1}, \ldots, v_{n} \mid[\bar{v}]\right)= & S_{2}\left(u_{1}, \ldots, u_{m} ; v_{1}, \ldots, v_{n}\right) \mid[\bar{v}] \\
& +\sum(-1)^{\mu^{\prime \prime}} S_{2}\left(u_{1}, \ldots, u_{m-1} ; v_{1}, \ldots, v_{n}\right) \mid u_{m} * \bar{v},
\end{aligned}
$$

where $(-1)^{\mu^{\prime}}$ and $(-1)^{\mu^{\prime \prime}}$ are the corresponding relative signs, then the following sum is 0 .

$(3)+(16)+(31),(10)+(21)+(28),(5)+(6)+(29)+(36),(11)+(12)+(27)+(35)$.

Since we also have

$$
\begin{aligned}
& S_{2}\left(u_{1}, \ldots, u_{m} ; v_{1}, \ldots, v_{n}\right) \\
& \quad=\sum(-1)^{\mu^{\prime}+\left|u_{1,1}\right|+\left|\bar{u}_{1,2}\right|+1} u_{1,1}\left|v_{1} * \bar{u}_{1,2}\right| S_{2}\left(u_{1,3}, \ldots, u_{m} ; v_{2}, \ldots, v_{n}\right) \\
& \quad=\sum(-1)^{\mu^{\prime \prime}} S_{2}\left(u_{1}, \ldots, u_{m-1} ; v_{1}, \ldots, v_{n, 1}\right)\left|u_{m} * \bar{v}_{n, 2}\right| v_{n, 3},
\end{aligned}
$$

where $(-1)^{\mu^{\prime}}$ and $(-1)^{\mu^{\prime \prime}}$ are the corresponding relative signs, then we have that the middle factor of the following sum is just the equality of $\mathbf{4}$ for $m=k-1$ and $n=l-1$,

$$
(7)+(17)+(20)+(25)+(32)+(40)+(26)+(41)+(30)+(38)+(39)
$$




$$
\begin{aligned}
& =\sum(-1)^{\mu} u_{1,1}\left|v_{1} * \bar{u}_{1,2}\right| \sum\left(u_{1,3}, \ldots, u_{m-1} ; v_{2}, \ldots, v_{n, 1}\right)\left|u_{m} * \bar{v}_{n, 2}\right| v_{n, 3} \\
& =0
\end{aligned}
$$

So the lemma is proved.

Proof of Theorem 2.4 We need only to construct a homotopy $T: \overline{V_{1} \otimes V_{2}} \rightarrow C(H)$ such that for all $u \in \bar{V}_{1}$ and $v \in \bar{V}_{2}, \bar{\Delta}(x)=\sum x^{\prime} \otimes x^{\prime \prime}$,

$$
\begin{aligned}
d T(u \otimes v) & +\sum \pi_{1}\left(u^{\prime}\right)\left|T\left(u^{\prime \prime} \otimes v\right)-\sum(-1)^{\left\langle u^{\prime \prime}, v\right\rangle+\left|u^{\prime}\right|+|v|} T\left(u^{\prime} \otimes v\right)\right| \pi_{1}\left(u^{\prime \prime}\right) \\
& +\sum(-1)^{\left\langle u, v^{\prime}\right\rangle} \pi_{2}\left(v^{\prime}\right)\left|T\left(u \otimes v^{\prime \prime}\right)-\sum(-1)^{|u|+\left|v^{\prime}\right|} T\left(u \otimes v^{\prime}\right)\right| \pi_{2}\left(v^{\prime \prime}\right) \\
& +\sum(-1)^{\left\langle u^{\prime \prime}, v^{\prime}\right\rangle} \pi_{1}\left(u^{\prime} ; \pi_{2}\left(v^{\prime}\right)\right) \mid T\left(u^{\prime \prime} \otimes v^{\prime \prime}\right) \\
& -\sum(-1)^{\left\langle u^{\prime \prime}, v\right\rangle+\left|u^{\prime}\right|+\left|v^{\prime}\right|} T\left(u^{\prime} \otimes v^{\prime}\right) \mid \pi_{2}\left(v^{\prime \prime} ; \pi_{1}\left(u^{\prime \prime}\right)\right) \\
= & \pi_{1}\left(u ; \pi_{2}(v)\right)-(-1)^{\langle u, v\rangle} \pi_{2}\left(v ; \pi_{1}(u)\right) .
\end{aligned}
$$

Suppose $\bar{\Delta}^{(n-1)}(x)=\sum x^{(1)} \otimes \cdots \otimes x^{(n)}$. Then define

$$
\tilde{T}(u \otimes v)=T(u \otimes v)+\sum_{n>1}(-1)^{\mu} T\left(u^{(1)} \otimes v^{(1)}\right)|\cdots| T\left(u^{(n)} \otimes v^{(n)}\right),
$$

where $(-1)^{\mu}$ is the sign of $\left(u^{(1)}, v^{(1)}, \ldots, u^{(n)}, v^{(n)}\right)$ relative to $\left(u^{(1)}, \ldots, u^{(n)}, v^{(1)}, \ldots, v^{(n)}\right)$, and then $\tilde{T}$ satisfies

$$
\begin{aligned}
d \tilde{T}(u & \otimes v)+\sum \pi_{1}\left(u^{\prime}\right)\left|\tilde{T}\left(u^{\prime \prime} \otimes v\right)-\sum(-1)^{\left\langle u^{\prime \prime}, v\right\rangle+\left|u^{\prime}\right|+|v|} \tilde{T}\left(u^{\prime} \otimes v\right)\right| \pi_{1}\left(u^{\prime \prime}\right) \\
& +\sum(-1)^{\left\langle u, v^{\prime}\right\rangle} \pi_{2}\left(v^{\prime}\right)\left|\tilde{T}\left(u \otimes v^{\prime \prime}\right)-\sum(-1)^{|u|+\left|v^{\prime}\right|} \tilde{T}\left(u \otimes v^{\prime}\right)\right| \pi_{2}\left(v^{\prime \prime}\right) \\
& +\sum(-1)^{\left\langle u, v^{\prime}\right\rangle} \pi_{2}\left(v^{\prime} ; \pi_{1}\left(u^{\prime}\right)\right) \mid \tilde{T}\left(u^{\prime \prime} \otimes v^{\prime \prime}\right) \\
& -\sum(-1)^{\left\langle u^{\prime \prime}, v^{\prime}\right\rangle+\left|u^{\prime}\right|+\left|v^{\prime}\right|} \tilde{T}\left(u^{\prime} \otimes v^{\prime}\right) \mid \pi_{1}\left(u^{\prime \prime} ; \pi_{2}\left(v^{\prime \prime}\right)\right) \\
= & \pi_{1}\left(u ; \pi_{2}(v)\right)-(-1)^{\langle u, v\rangle} \pi_{2}\left(v ; \pi_{1}(u)\right) .
\end{aligned}
$$

Notice that by the definition in Theorem 2.1, we have

$$
\begin{aligned}
& \pi_{1}\left(u ; \pi_{2}(v)\right)=S\left(\pi_{1}(u) ; \pi_{2}(v)\right)+\sum_{n>1} S\left(\pi_{1}\left(u^{(1)}\right), \ldots, \pi_{1}\left(u^{(n)}\right) ; \pi_{2}(v)\right), \\
& (-1)^{\langle u, v\rangle} \pi_{2}\left(v ; \pi_{1}(u)\right)=S^{\prime}\left(\pi_{1}(u) ; \pi_{2}(v)\right)+\sum_{n>1} S^{\prime}\left(\pi_{1}(u) ; \pi_{2}\left(v^{(1)}\right), \ldots, \pi_{2}\left(v^{(n)}\right)\right),
\end{aligned}
$$

where $S, S^{\prime}$ are as defined in Lemma 2.1. Let $S_{2}$ be as defined in Lemma 2.1. Then we have

$$
\begin{aligned}
& S_{2}\left(\pi_{1}\left(u^{(1)}\right), \ldots, d \pi_{1}\left(u^{(s)}\right), \ldots, \pi_{1}\left(u^{(m)}\right) ; \pi_{2}\left(v^{(1)}\right), \ldots, \pi_{2}\left(v^{(n)}\right)\right) \\
& \quad=(-1)^{\left|u^{(s)}\right|+1} S_{2}\left(\pi_{1}\left(u^{(1)}\right), \ldots, \pi_{1}\left(u^{(s)}\right) \mid \pi_{1}\left(u^{(s+1)}\right), \ldots, \pi_{1}\left(u^{(m)}\right) ; \pi_{2}\left(v^{(1)}\right), \ldots, \pi_{2}\left(v^{(n)}\right)\right), \\
& S_{2}\left(\pi_{1}\left(u^{(1)}\right), \ldots, \pi_{1}\left(u^{(m)}\right) ; \pi_{2}\left(v^{(1)}\right), \ldots, d \pi_{2}\left(v^{(t)}\right), \ldots, \pi_{2}\left(v^{(n)}\right)\right) \\
& \quad=(-1)^{\left|v^{(t)}\right|+1} S_{2}\left(\pi_{1}\left(u^{(1)}\right), \ldots, \pi_{1}\left(u^{(m)}\right) ; \pi_{2}\left(v^{(1)}\right), \ldots, \pi_{2}\left(v^{(t)}\right) \mid \pi_{2}\left(v^{(t+1)}\right), \ldots, \pi_{2}\left(v^{(n)}\right)\right) .
\end{aligned}
$$

So by Lemma 2.1, we may define

$$
\tilde{T}(u \otimes v)=\sum_{m, n \geq 1} S_{2}\left(\pi_{1}\left(u^{(1)}\right), \ldots, \pi_{1}\left(u^{(m)}\right) ; \pi_{2}\left(v^{(1)}\right), \ldots, \pi_{2}\left(v^{(n)}\right)\right) .
$$

The theorem is proved.

Now suppose for $i=1,2, \ldots, n, n>1$, we have well defined trivial Massey-product $\left\langle\alpha_{i, 1}, \ldots, \alpha_{i, s_{i}}\right\rangle$ on the cobar complex $C(H)$. Then we have $n$ realizations $\pi_{i}: \bar{M}_{s_{i}} \rightarrow C(H)$ defined by letting $\pi_{i}\left(e_{k, k+l}\right)=\overline{\left\langle\alpha_{i, k}, \ldots, \alpha_{i, l}\right\rangle}$ for all $k$ and $l$, where $M_{n}$ is the Massey coalgebra defined in Section 1. Then, we have a new multi-product

$$
\left\langle a ; \alpha_{1,1}, \ldots, \alpha_{1, s_{1}} ; \ldots ; \alpha_{n, 1}, \ldots, \alpha_{n, s_{n}}\right\rangle=\left\langle a ;\left(\pi_{1} \otimes \cdots \otimes \pi_{n}\right)\left(e_{1,1+s_{1}} \otimes \cdots \otimes e_{1,1+s_{n}}\right)\right\rangle .
$$

Specifically, when all $s_{i}=1$, the product is just the new Massey product defined in [1]. 
Acknowledgements The author here expresses his deep gratitude to the referee for his helpful advices.

\section{References}

[1] Zheng, Q. B.: A New Massey Product on Ext Groups. Journal of Algebra, 183, 378-395 (1996)

[2] May, J. P.: A general algebraic approach to Steenrod algebra. Lecture Notes in Mathematics, 168, 153-231

[3] Adams, J. F.: On the structure and application of the Steenrod algebra. Math. Helv., 32, 180-247 (1958)

[4] Massey, W. S.: Some higher order cohomology operations. Sympos. Internat. Alg. Top., Mexico City, $145-154(1958)$

[5] May, J. P.: Matric Massey products. Journal of Algebra, 12, 533-568 (1969)

[6] May, J. P.: The cohomology of restricted Lie algebra and of Hopf algebra. Journal of Algebra, 3, 123-146 (1966)

[7] MacLane, S.: Homology, Springer Verlag, Berlin and New York, 1963

[8] Zheng, Q. B.: S-module and the New Massey-Product. Journal of Algebra, 190, 487-497 (1997) 